\title{
Finding the radial parallel temperature profile in a non-neutral plasma using equilibrium calculations on experimental data
}

\author{
Grant W. Hart \\ grantwhart@gmail.com \\ Bryan G. Peterson \\ bryan_peterson@byu.edu
}

Follow this and additional works at: https://scholarsarchive.byu.edu/facpub

Part of the Astrophysics and Astronomy Commons, and the Physics Commons

\section{Original Publication Citation}

Hart, Grant W. and Bryan G. Peterson."Finding the radial parallel temperature profile in a nonneutral plasma using equilibrium calculations on experimental data." Physics of Plasmas 13 (26).

\section{BYU ScholarsArchive Citation}

Hart, Grant W. and Peterson, Bryan G., "Finding the radial parallel temperature profile in a non-neutral plasma using equilibrium calculations on experimental data" (2006). Faculty Publications. 325.

https://scholarsarchive.byu.edu/facpub/325

This Peer-Reviewed Article is brought to you for free and open access by BYU ScholarsArchive. It has been accepted for inclusion in Faculty Publications by an authorized administrator of BYU ScholarsArchive. For more information, please contact ellen_amatangelo@byu.edu. 


\title{
Finding the radial parallel temperature profile in a non-neutral plasma using equilibrium calculations on experimental data
}

\author{
Grant W. Hart and Bryan G. Peterson \\ Department of Physics and Astronomy, Brigham Young University, Provo, Utah 84602
}

(Received 23 November 2005; accepted 22 December 2005; published online 8 February 2006)

\begin{abstract}
In 1992, Eggleston et al. [D. L. Eggleston et al., Phys. Fluids B 4, 3432 (1992)] reported on a technique for measuring the radial temperature profile in a pure-electron plasma confined in a Malmberg-Penning trap by partially dumping the plasma onto a charge collector at the end of the trap. For short plasmas and short confining rings, the assumptions in their paper are violated and a more general calculation is needed. This paper presents a variation of the standard equilibrium calculation to find the temperature profile of a pure-electron plasma. Eggleston's shortcut "evaporation" temperature method is found to require a correction factor that can be calculated using methods described in this paper. For typical conditions, the evaporation method overstates the actual temperature by a factor ranging from 1.1 to 1.5 or more, depending on the plasma's total charge and temperature and the geometry of the trap. (C) 2006 American Institute of Physics.
\end{abstract}

[DOI: $10.1063 / 1.2167586]$

\section{INTRODUCTION}

When analyzing the behavior of a plasma, the two most fundamental parameters are the plasma density and temperature. Pure electron plasmas have special challenges in making these measurements because of their very low density, typically in the $10^{12} \mathrm{~m}^{-3}$ range. This means that most standard density and temperature diagnostics, such as Langmuir probes, interferometry, and Thomson scattering, are impractical. Pure ion plasmas, on the other hand, can be diagnosed with laser-induced fluorescence, at least for selected ion species.

Pure electron plasmas are typically confined in Malmberg-Penning traps. ${ }^{1}$ In these traps, the non-neutral plasma is radially confined by an applied axial magnetic field and axially confined by electrostatic potentials applied to rings at the ends of these cylindrical traps. This is illustrated in Fig. 1. These traps typically operate in a fill-manipulatedump cycle, as described in Sec. II.

The density in one of these traps is typically measured destructively by collecting the charge at various radial positions as the plasma is dumped out the end of the trap. The charge can be collected on either a single charge collector, Faraday cups behind holes in the end plate, ${ }^{2}$ a set of concentric rings, ${ }^{3,4}$ or a two-dimensional phosphor screen. ${ }^{5}$ Whatever device is used, this process gives the integral of the density along the field lines. The density as a function of $z$ (the direction of $\mathbf{B}$ ) is then calculated using an assumption of electrostatic equilibrium and the known geometry of the device. 3,6

The temperature profile is a more difficult quantity to measure. The earliest temperature measurements in a Malmberg-Penning trap involved measuring the dispersion relation of electrostatic waves in the plasma. ${ }^{7}$ This technique gives an average measurement for the whole plasma and involves careful wave propagation measurements. The perpendicular temperature (involving velocity perpendicular to the magnetic field) can be measured by a "magnetic beach analyzer." 8 The perpendicular temperature is normally assumed to be closely related to the parallel temperature. More recently, both the frequencies ${ }^{4}$ and thermally exited amplitudes $^{9}$ of normal modes of oscillation have been proposed or used to measure the parallel temperature. Again, these measure an average temperature of the whole plasma.

In 1992, Eggleston et al. ${ }^{10}$ published a method of measuring the radial parallel temperature profile. This method works well if several assumptions are met. It also gave a shortcut method that allowed a simple measurement of the on-axis temperature. This paper is a generalization of Eggleston's method that is not restricted by the assumptions of that method. In particular, it allows the measurement of the temperature for short plasmas and devices with short confining rings. A correction factor is found that can be applied to the simple on-axis temperature measurement.

In this paper, we first discuss Eggleston's method and the assumptions involved in it. The next section discusses equilibrium calculations and the modifications that are necessary in order to correctly model a plasma that has been partially dumped. In the next sections, we apply this method to data from a particle-in-cell (PIC) simulation and to data taken from our experiment. In the final sections, we discuss the necessary corrections to the simplified on-axis measurement and draw our final conclusions.

\section{EGGLESTON'S METHOD AND ITS ASSUMPTIONS}

This method is applied to a single-species plasma confined in a Malmberg-Penning trap operated in a fill-trapmanipulate-dump cycle. The electrons are thermionically emitted from a spiral filament (shown on the left in Fig. 1.) The left-side ring is grounded and the electrons are allowed to stream into the confinement region. After some time the ring voltage is again changed to its confining value. The next step is to perform whatever experiment is desired on the confined plasma, and at the end of that time the plasma is dumped onto the charge collectors on the right by grounding 


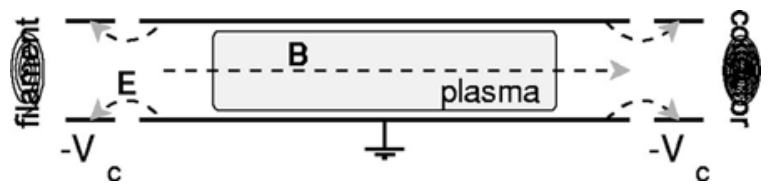

FIG. 1. Electric and magnetic fields in a Malmberg-Penning trap.

the right-side ring, allowing the electrons to escape along the field lines until they impact and are absorbed by the positively biased charge collectors. In our experiment, these charge collectors are a set of 10 concentric disks mounted in a conical fashion, so that each disk collects all the charge that impacts it between its outer radius and the radius of the next smaller disk. See Fig. 2. Three of the disks are split to allow measurement of up/down and right/left asymmetries in the plasma. There were no such asymmetries in this set of experiments. The disks are connected to a set of charge integrators, allowing measurement of the total charge collected as a function of radius. With this setup, there is no information on the timing of the arrival of the charge.

To implement Eggleston's method, the magnitude of the voltage on one of the confining rings is dropped to a value intermediate between the full voltage and ground and the escaped charge is collected on the charge collectors. After that measurement is made, the remainder of the plasma is dumped and a new plasma is prepared. This new plasma can be dumped at a different intermediate voltage. The entire data set consists of the set of radial profiles of charge lost as a function of voltage applied on the confinement ring. Clearly this measurement requires reproducibility in the plasma. Our shot-to-shot variability for density variations is less than $1 \%$.

The potential as a function of $z$ along the $r=0$ axis for one of our typical plasmas is shown in Fig. 3. This is taken from a standard equilibrium calculation, but inverted to appear as a potential energy diagram for electrons. The plasma creates a substantial self-potential, $\phi_{c}$, in the center. Only the particles that have enough energy to get over the potential hill $\phi_{\max }$ will escape. Therefore, the critical parameter to know in the analysis of this data set is $\phi_{h}=\phi_{\max }-\phi_{c}$. Measurement of charge lost versus $\phi_{h}$ will give us the temperature. All the particles in the plasma pass through the midplane and in the absence of collisions have a constant energy. This means that the fraction of the plasma lost for a given $\phi_{h}$ is just the fraction of the particles with total energy greater than $q \phi_{h}$ (relative to the middle of the plasma), which for a Maxwellian velocity distribution is just $\operatorname{erfc}\left(\sqrt{q \phi_{h} / k T}\right)$,

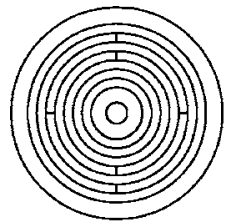

Front View

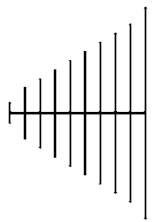

Side View
FIG. 2. Charge collection disks used in our experiment. Three of the rings are split to check for up/down and right/left asymmetries in the plasma.

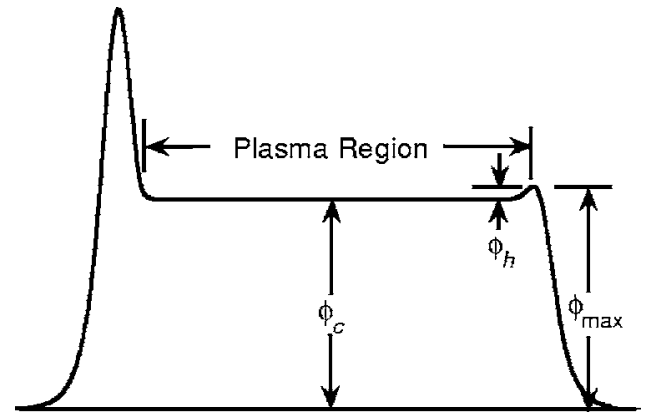

FIG. 3. Potential energy curve for a partially dumped electron plasma. The different potentials involved in the dump are indicated on the plot.

where the erfc function is the complimentary error function of statistics.

In his calculation of $\phi_{h}$, Eggleston made several approximations that were justified in his context, but which do not work in our experiment. The first approximation had to do with $\phi_{c}$. He approximated the plasma as a flat-ended cylinder of known length. The total $z$-integrated charge as a function of radius is then divided by $2 \pi r L$, where $r$ is the radial position and $L$ is the assumed plasma length, to produce an approximation for the density of the plasma at the midplane. Assuming axisymmetry and $L$ much longer than the wall radius, this can be integrated to find $\phi_{c}(r)$, using the equation

$$
\frac{1}{r} \frac{d}{d r}\left(r \frac{d}{d r} \phi_{c}(r)\right)=-\frac{q}{\epsilon_{0}} n_{r}(r)
$$

where $n_{r}(r)$ is the density of plasma remaining after the partial dump and $q$ is the charge on a single particle.

In reality, the plasma extends some distance up the potential hill, with the amount of that extent depending on the radial position, the temperature, and the amount of charge at that radius. Eggleston recognized that this limited the applicability of his method to long plasmas, since the extra extent of the plasma will not change the value of $n_{r}(r)$ significantly if the plasma is long.

The second approximation is that the plasma does not significantly affect the value of the potential hill, $\phi_{\max }$. This is a more problematic approximation, since by definition the plasma is close to the confining ring while it is being dumped. The importance of this approximation depends on the density and temperature of the plasma and the length of the confining ring. If the $z$ extent of the confining ring is greater than its radius, then this approximation is better. On the other hand, if the confining ring is short compared to its radius, then the plasma will have a significant effect on $\phi_{\max }$. Figure 4 shows the effect of the plasma on both the height and position of $\phi_{\max }$ for our experiment, which has a confining ring that is $2.5 \mathrm{~cm}$ long and $4.0 \mathrm{~cm}$ in radius.

The confining potential $\phi_{\max }$ is made up of two parts: the vacuum field contribution, $\phi_{v}$, and the plasma contribution, $\phi_{p}$. The vacuum contribution depends on the radius and the potential applied to the confining ring. We can write this as $\phi_{v}(r)=\alpha(r) \phi_{r}$, where $\phi_{r}$ is the potential on the confinement 


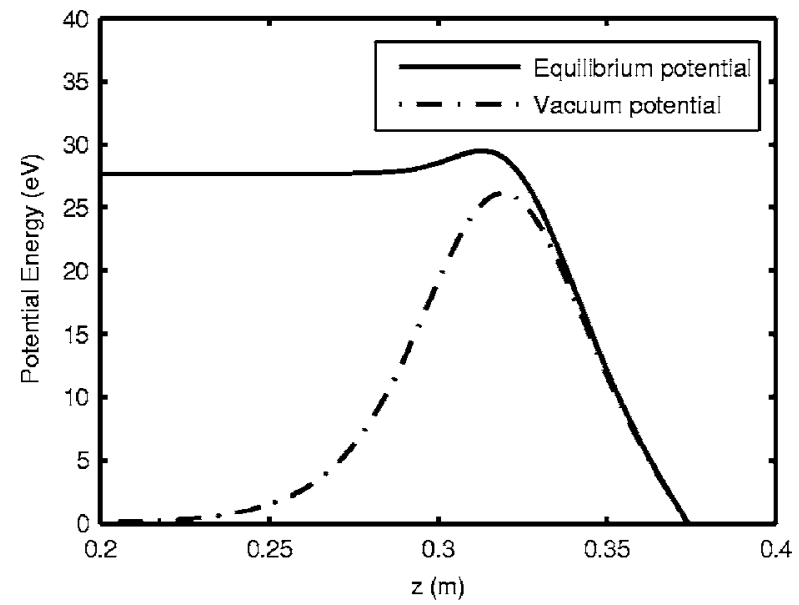

FIG. 4. The potential energy as a function of $z$ with and without plasma in the trap for our geometry. Note that both the size and position of the potential hill are affected by the presence of the plasma.

ring and $\alpha(r)$ is the ratio of the maximum potential at radius $r$ to the ring potential. This means that $\phi_{h}$ can be written as

$$
\phi_{h}=\phi_{\max }-\phi_{c}=\alpha(r) \phi_{r}+\phi_{p}-\phi_{c} .
$$

Finding the temperature of the plasma at each radius boils down to finding the mapping between the potential applied to the confinement ring and the size of the potential hill at each radius and then fitting the curve of charge lost versus confining potential hill to the function $\operatorname{erfc}\left(\sqrt{q \phi_{h} / k T}\right)$ and obtaining the temperature from the fitting coefficient. In order to calculate $\phi_{h}$ for use as the $x$ axis of this fit, we need to calculate both $\phi_{c}$ and $\phi_{\max }$. This requires an equilibrium calculation, as discussed in the next section.

The most commonly used result in Eggleston's paper is the shortcut method that he gave to determine the central temperature of the plasma. This technique involves doing a slow dump of the plasma and looking only at the charge that escapes on the axis of the experiment. The charge escapes at the center first because the plasma potential is highest there and the vacuum potential is lowest. Expanding the erfc function in the tail of the distribution at that position, Eggleston found that

$$
\frac{d\left(\ln Q_{\mathrm{esc}}\right)}{d\left(q \phi_{h}\right)}=\frac{-1.05}{k T_{\|}}
$$

to about $5 \%$ accuracy. This calculation still depends on $\phi_{h}$ rather than $\phi_{r}$, but the two assumptions mentioned above make the calculation easier, since under conditions where those assumptions hold, both $\phi_{c}$ and $\phi_{p}$ will be roughly constant as the ring potential $\phi_{r}$ changes. This means that at the center $d\left(q \phi_{h}\right) \approx d\left(q \phi_{v}\right)=\alpha(0) d\left(q \phi_{r}\right)$, allowing the simple mapping between change in the ring voltage and the change in the potential hill. This makes the fitting equation approximately

$$
k T=\frac{-1.05 \alpha(0)}{\left(\frac{d\left(\ln Q_{\mathrm{esc}}\right)}{d\left(q \phi_{r}\right)}\right)},
$$

where the derivative is calculated by a fit to the data. This result works well when the assumptions hold, but must be modified when they do not. Because of the slow dump and escape of the plasma, the result of this temperature measurement is often referred to as the evaporation temperature.

\section{EQUILIBRIUM CALCULATIONS FOR BARELY CONFINED PLASMAS}

The standard equilibrium calculation starts with the assumption that for a plasma in electrostatic equilibrium along the magnetic-field lines, the distribution function will be of the form

$$
\begin{aligned}
f(v, r, z)= & n_{0}(r) \sqrt{\frac{m}{2 \pi k T(r)}} \exp \left(-\frac{E}{k T(r)}\right) \\
= & n_{0}(r) \sqrt{\frac{m}{2 \pi k T(r)}} \\
& \times \exp \left(-\frac{1 / 2 m v^{2}+q\left[\phi(r, z)-\phi_{c}(r)\right]}{k T(r)}\right),
\end{aligned}
$$

where $n_{0}(r)$ is the density profile in the midplane of the plasma and $\phi_{c}(r)$ is the potential profile in the midplane. We assume axisymmetry. The presence of $T(r)$ in the formula above shows that this is not a full thermal equilibrium, where everything would be at the same temperature, but rather a pseudoequilibrium where the system has come to equilibrium in the axial $z$ direction but the much slower equilibration in the radial $r$ direction through collisions and viscosity has not yet taken place. When this distribution is integrated over all velocities, we get the density distribution

$$
n(r, z)=n_{0} \exp \left(-\frac{q\left[\phi(r, z)-\phi_{c}(r)\right]}{k T(r)}\right) .
$$

An equilibrium code combines this equation with Poisson's equation and solves for the self-consistent density and potential, given the constraint that the integral of the charge density over $z$ must match the measured values,

$$
Q(r)=q \int n(r, z) d z .
$$

Our standard equilibrium code EQUILSOR has been described elsewhere. 6

However, this calculation needs correction if the plasma has been partially dumped: all particles with a velocity greater than that necessary to escape over the potential hill will be gone. At a location where the potential is $\phi(r, z)$, the maximum velocity for a confined particle will be

$$
v_{\mathrm{esc}}=\sqrt{\frac{2 q\left[\phi_{\max }(r)-\phi(r, z)\right]}{m}},
$$

where $\phi_{\max }(r)$ is the maximum potential hill at each radial position $r$. Our distribution function is therefore a Maxwellian truncated at velocity $v_{\text {esc }}$. Again, this is only a pseu- 
doequilibrium, since collisions will cause modification of the distribution function, but on a time scale short compared to a collision time it should be correct. In our experiment, the collision time is several milliseconds, and the dump takes place in less than $20 \mu \mathrm{s}$. When the distribution function is integrated from $-v_{\text {esc }}$ to $v_{\text {esc }}$, rather than the result in Eq. (6) we get

$$
\begin{aligned}
n(r, z)= & n_{0} \exp \left(-\frac{q\left[\phi(r, z)-\phi_{c}(r)\right]}{k T(r)}\right) \\
& \times \operatorname{erf}\left(\sqrt{\frac{q\left[\phi_{\max }(r)-\phi(r, z)\right]}{k T(r)}}\right) .
\end{aligned}
$$

The erf part of this expression is most important at the ends. This is because at the ends the potential is approaching $\phi_{\max }$ and the low velocity (in the midplane) particles have already been reflected back toward the center of the plasma, making the fraction that has been lost more significant. Note that the plasma density at the potential peak will be identically zero with this expression, because $\phi_{\max }(r)=\phi(r, z)$ at the peak, by definition, so all particles that had enough energy to reach that point will escape. Using our PIC code RATTLE ${ }^{12}$ to model a partial dump, we have verified that the plasma remaining in the trap is closely approximated by this expression.

The equilibrium calculation for a partially dumped plasma is therefore modified to use Eq. (9) instead of Eq. (6) and otherwise proceeds as before, using the same constraint on the integral of the density. This expression actually can be used for any equilibrium calculation, because full confinement would require an infinite potential hill, which is unphysical. For a well confined plasma the difference between Eqs. (6) and (9) is negligibly small, however, since the height of the potential hill is many times $k T$.

\section{TEMPERATURE CALCULATION WITH THE EQUILIBRIUM CODE}

The data set for a temperature calculation consists of profiles of charge lost versus voltage applied to the confinement ring. A complication in doing the equilibrium calculation to find $\phi_{h}$ is that it is necessary to know the temperature as a function of radius to do the calculation. This, of course, is unknown because it is what we are trying to find. To start the calculation, we use the idea behind the evaporation temperature, but at each radius, using the appropriate $\alpha$ for that radius. This does not give the correct temperature, but it is close enough to get started. It should be pointed out that the equilibrium calculation is nonlocal in that the size of the potential hill at one radius is affected by the temperature and density at other radii, since the potential depends on the distribution of all the charge.

There are two ways to use the data set to find the temperature. One is to use the fraction of the plasma that is lost at each radius for some ring voltage and adjust the temperature at each radius during the equilibrium solve so that the appropriate fraction is lost. In practice it is necessary to do this at a series of voltages because the plasma at different radii does not escape at the same potential due to the radial variations in the confining voltage and midplane potential. The confining potential goes up as a function of radius and the central potential drops. This requires an iterative procedure where the evaporation temperature is used as a first guess, the temperature as a function of radius is calculated, which is used as a second guess, and the temperature is calculated again. Usually only two iterations are required. Our experience with this method is that it is very sensitive to experimental uncertainties. When used with real data, it often gave results that were inconsistent at different ring voltages.

The second method uses more of the data provided in the data set. With this method we use the temperature estimates discussed at the start of this section and calculate an equilibrium for each ring voltage in the data set and store $\phi_{h}$ as a function of $r$ for each one. The charge lost at each radius can then be fit (using a nonlinear least-squares fit) to the function

$$
q_{\text {lost }}=C \operatorname{erfc}\left(\sqrt{\frac{q \phi_{h}}{k T}}\right),
$$

where $C$ and $k T / q$ are the unknown parameters. If the particles in the plasma are electrons, $k T / q$ is just the temperature in $\mathrm{eV}$. We have tried iterating on the results as in the first method, but it is found that the results are relatively insensitive to the initial guess of the temperature (as long as it is in the ballpark) and the iteration results in a negligible change in the final results.

In doing the fit, the question arises as to how much of the data should be used in the fit. There are experimental difficulties in accurately measuring the charge dumped when a large fraction of the plasma comes out. Our electronics integrates the charge that comes out during a $10 \mu \mathrm{s}$ window. This works very well for most of the dumps, but when we approach the zero velocity portion of the Maxwellian distribution, not all of the slow charge will have a chance to escape. There are also dynamics associated with the dump when it is deep ${ }^{11}$ that may affect the amount of charge dumped beyond the simple picture given here. We deal with this by doing the fit repeatedly on the data, including more data each time. We start by doing a fit using the data up to $1 \%$ of the plasma lost and continue making fits until we reach the point where $50 \%$ of the plasma is lost. We then choose the fit that has smallest mean-square error per significant data point. By significant data point we mean a data point representing more than $1 \%$ of the plasma lost. This procedure usually results in the fit extending to somewhere between 10 and $25 \%$ of the plasma lost. By using the data this way we use more of the information in the data than with the first method and reduce the uncertainty associated with experimental error.

\section{COMPARISON WITH SIMULATION RESULTS}

We first tested this measurement with data taken from RATTLE. We did this because with a simulation we know what the actual temperature distribution is and can compare it to the analysis results. For our simulation we used a quadratic temperature profile with a central temperature of $1 \mathrm{eV}$ and an outer temperature of $3.5 \mathrm{eV}$. We chose this profile because that covers a range of temperatures that is fairly 


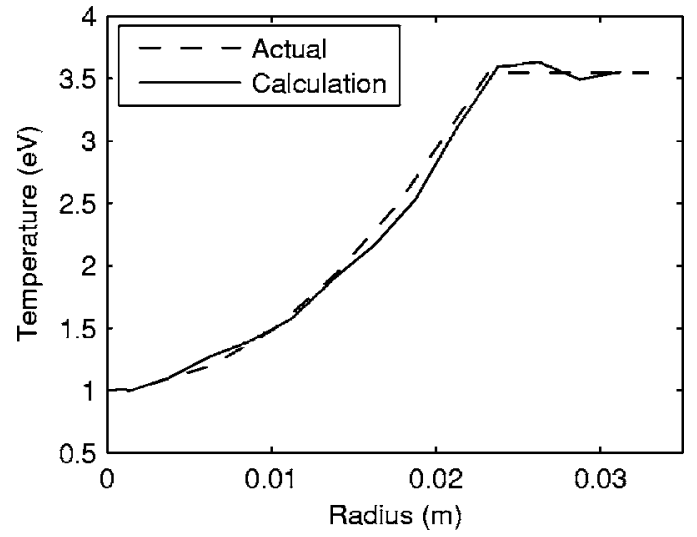

FIG. 5. The actual and measured temperatures from our simulation. The dashed line is the actual temperature and the solid line is the result from the equilibrium calculation.

typical of those we see in our plasmas (although not necessarily at the same time) and we wanted to determine if the method could handle significant gradients correctly. RATTLE is an axisymmetric $r-z$ PIC code where the particles can only move along the field lines. For each run we started with the plasma in equilibrium and then changed the end potential to the desired intermediate value. We used experimentally measured time histories of the voltage on the rings so that the simulation would closely correspond to the experimental situation. The output for each simulation was the charge lost as a function of radius and time. We did runs with the ring voltage dropping to $-100 \mathrm{~V},-97.5 \mathrm{~V},-95.0 \mathrm{~V}, \ldots$, $-2.50 \mathrm{~V}, 0 \mathrm{~V}$. The profiles of total charge lost as a function of radius and voltage were then used as inputs to the equilibrium code calculation discussed in the section above.

One complication in the simulation results was the continued evaporation of simulation particles over the potential hill after the potential stopped changing. The magnitude of the evaporation was not physical; it is due to the fact that we were only able to run with $\sim 1 \times 10^{6}$ simulation particles, instead of the $\sim 5 \times 10^{9}$ actual particles in the experiment. This created large density and potential fluctuations that scattered the particles in velocity space and sent some of them over the potential hill. The magnitude of this evaporation decreased approximately as $1 / N$, where $N$ is the number of particles in the simulation. To compensate for this effect, we ran the simulation for a long time after the ring voltage had completed its change, measured the evaporation rate, and subtracted that from the data. This allowed a more accurate estimate of the charge that would be lost in the actual experiment.

Results of applying our equilibrium calculation method to these data are shown in Fig. 5. The dashed line is the actual temperature distribution and the solid line is the calculated temperature. As can be seen, they agree very well, giving confidence that this method should work with real data.

\section{USE WITH REAL DATA}

Our experiment is a fairly typical Malmberg-Penning trap with a nominal plasma length of $60 \mathrm{~cm}$ and a ring radius

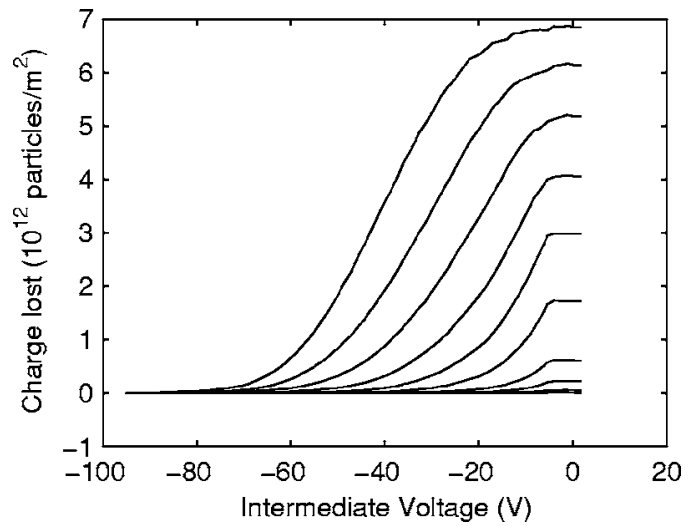

FIG. 6. A typical data set showing the amount of charge dumped as a function of voltage on the confining ring. Each curve represents charge collected at a different radius. Starting at the top curve, the radii are at 0.35 , $0.85,1.15,1.45,1.75,2.05,2.35,2.6505,2.95$, and $3.50 \mathrm{~cm}$. The raw data are scaled by the area of the charge collectors so that they represent $\int n d z$. These data were taken at $0.125 \mathrm{~s}$ after the plasma was trapped.

of $4 \mathrm{~cm}$. Typically our plasmas had a radius of about $2.5 \mathrm{~cm}$. The central density in these data is near $7 \times 10^{12} \mathrm{~m}^{-3}$. Our neutral gas pressure is normally near $8 \times 10^{-9}$ Torr. While we have not made the measurement for these specific data sets, a typical particle confinement time in this machine is $5-6 \mathrm{~s}$.

As discussed in Sec. II, a data set consists of measurements of the charge dumped at various radii for different voltages applied to the confinement ring. We measured both the charge that came out when the voltage was changed to its intermediate value and also the charge that came out later when the voltage was changed to ground from the intermediate value. We checked our data for consistency between these two measurements by summing them at a particular voltage and verifying that we had accounted for all the charge that was originally present in the plasma. A typical data set is shown in Fig. 6. Each curve in this plot represents charge collected at a different radius. The charge data are divided by $e$ and the area of the detector at that radius so that it represents $\int n d z$.

The results of analysis of this data set and two others are shown in Fig. 7. The three data sets are taken at different times in the evolution of the plasma: 0.125, 0.255, and $0.500 \mathrm{~s}$ after the trapping of the plasma. The solid curves in the plot represent a fit of the measurements to a curve of the form $a+b x^{2}$. We do this because of the $\sim 5 \%$ uncertainty in the measurements and the requirement that the derivative of the temperature with respect to $r$ must be zero at $r=0$. We can see that the temperature of the plasma is dropping with time and that the temperature gradient is small at all times. We expect the outer edge to be somewhat warmer than the inside because expansion of the plasma column leads to conversion of electrostatic potential energy into thermal energy. ${ }^{13}$ The fact that expansion of the plasma is a heating mechanism makes the cooling of the plasma at the same time as it is expanding somewhat puzzling. We think it is likely due to inelastic collisions with neutral gas in the trap, particularly since a higher neutral pressure leads to colder plasmas as well as larger temperature gradients in our machine. 


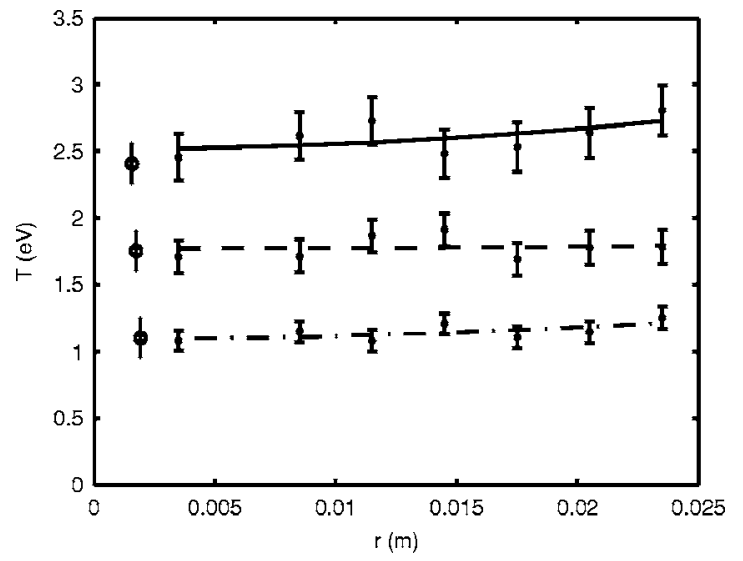

FIG. 7. The results of the calculation on three data sets taken at three different times in the evolution of our plasma. The solid line is for $t$ $=0.125 \mathrm{~s}$, the dashed line for $t=0.255 \mathrm{~s}$, and the dot-dashed line is for $t$ $=0.500 \mathrm{~s}$. Also shown for comparison to the left of the curves and marked with circles are evaporation temperatures taken on the same plasmas, corrected by the factor discussed in Sec. VII.

The higher gradient shows that radial diffusion has increased, but the lower temperature shows that energy is being absorbed by the neutral gas.

\section{CORRECTION OF EVAPORATION TEMPERATURE MEASUREMENT}

To the left of the curves in Fig. 7 there are three points that are marked with circles. These are corrected evaporation temperature measurements taken at the same times $(0.125$, 0.255 , and $0.500 \mathrm{~s}$ ) in the evolution of the plasma as the other data. If we just take the temperature measurements as specified in Eq. (4), we find that the temperatures measured are significantly higher (a factor of 1.3-1.5 higher) than the measurements reported here. To resolve this discrepancy, we examined the assumptions of Eggleston's theory in our situation. We found that both assumptions were violated, and the violation of the second was about twice as big as the violation of the first in our case. Figure 8 shows $d \phi_{h} / d \phi_{r}$ as a function of the fraction of the plasma that was lost, derived from the equilibrium calculation. Clearly, this slope never equals $\alpha(0)$, the value predicted by Eggleston's model

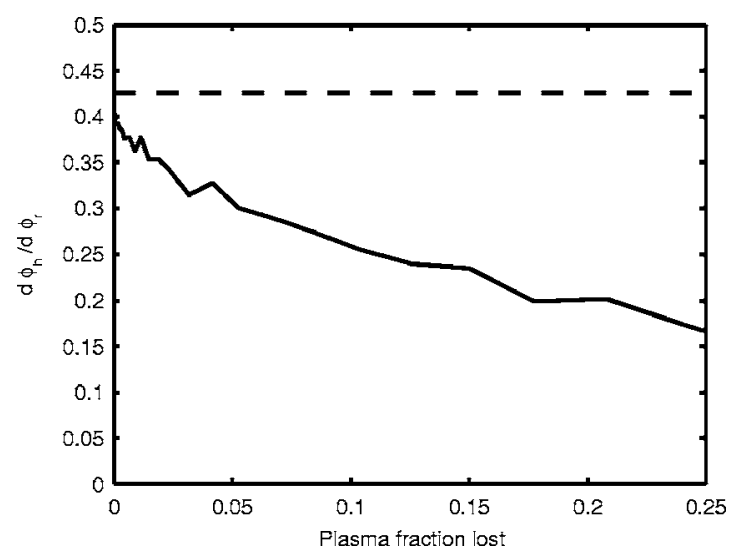

FIG. 8. $d \phi_{h} / d \phi r$ plotted as a function of fraction of central plasma lost. The dashed line at the top is $\alpha(0)$. Even at zero plasma loss, these do not agree. (shown by the dashed line in the figure) even at zero plasma loss. Taking the derivative of Eq. (2) gives us

$$
\frac{d \phi_{h}}{d \phi_{r}}=\alpha(0)+\frac{d \phi_{p}}{d \phi_{r}}-\frac{d \phi_{c}}{d \phi_{r}} .
$$

As the ring potential is dropped, the plasma starts to expand, even before plasma starts to be lost. This causes two effects. First, the central potential drops because the central density drops. This makes $d \phi_{c} / d \phi_{r}$ a positive number. Eggleston discussed this and chose to ignore it because it is only a few percent effect [in our case $4 \%-5 \%$ of $\alpha(0)$ just as the plasma starts to escape]. The second effect is that the plasma moves closer to the point of peak potential. As it does so, it makes more significant contributions to the potential at that point, as can be seen in Fig. 4. This makes $d \phi_{p} / d \phi_{r}$ a negative number. In our plasma it is about $8 \%$ of $\alpha(0)$ at the point where the plasma is just starting to be lost. These two effects (the central potential dropping and the maximum potential being raised) combine to make the change in the hill potential less than you would calculate from the change in vacuum potential alone, which makes the temperature calculated from Eq. (4) too high.

To quantify the density and temperature dependence of this correction factor, we modified the equilibrium code to calculate the fraction that would remain in the plasma for a given ring voltage. During the convergence of the equilibrium calculation, the program calculates the height of the confining hill and modifies the $\int n d z$ constraint so that the proper fraction of the plasma is confined. Calculating equilibria for multiple confining potentials allows calculation of $d \phi_{h} / d \phi_{r}$. Looking at Fig. 8 makes it clear that the correction factor depends on how much plasma has been dumped. We calculate the correction factor to use in analyzing experimental data by calculating the quantity

$$
\langle c\rangle=\left\langle\frac{\alpha(0)}{\frac{d \phi_{h}}{d \phi_{r}}}\right\rangle,
$$

where the brackets indicate an average over the range of a fraction of central plasma lost from 0.005 to 0.1 . These limits represent a typical range for collecting evaporation data. The lower limit comes from the fact that our digital oscilloscope has an 8 bit resolution, so if the entire charge dumped from the center of the plasma fills the screen, we cannot see a change of less than one part in 256 of that charge. The upper limit comes from the fact that a plot of the log of the collected charge versus ring voltage starts to deviate from linearity in the neighborhood of $10 \%$ of the central charge lost.

The result of these calculations for the profile of the $125 \mathrm{~ms}$ data is shown in Fig. 9. Part (a) shows theoretical curves of the correction factor as a function of total particle number for different temperatures. Note that higher particle number and lower temperature tend to make the correction factor larger and that at zero particles it goes to 1 , as expected. Clearly we would expect more plasma to increase the plasma effect at the ring. The effect of a lower temperature is also reasonable, since a hotter plasma is more diffuse at the ends and so the effect of the plasma on the potential hill 

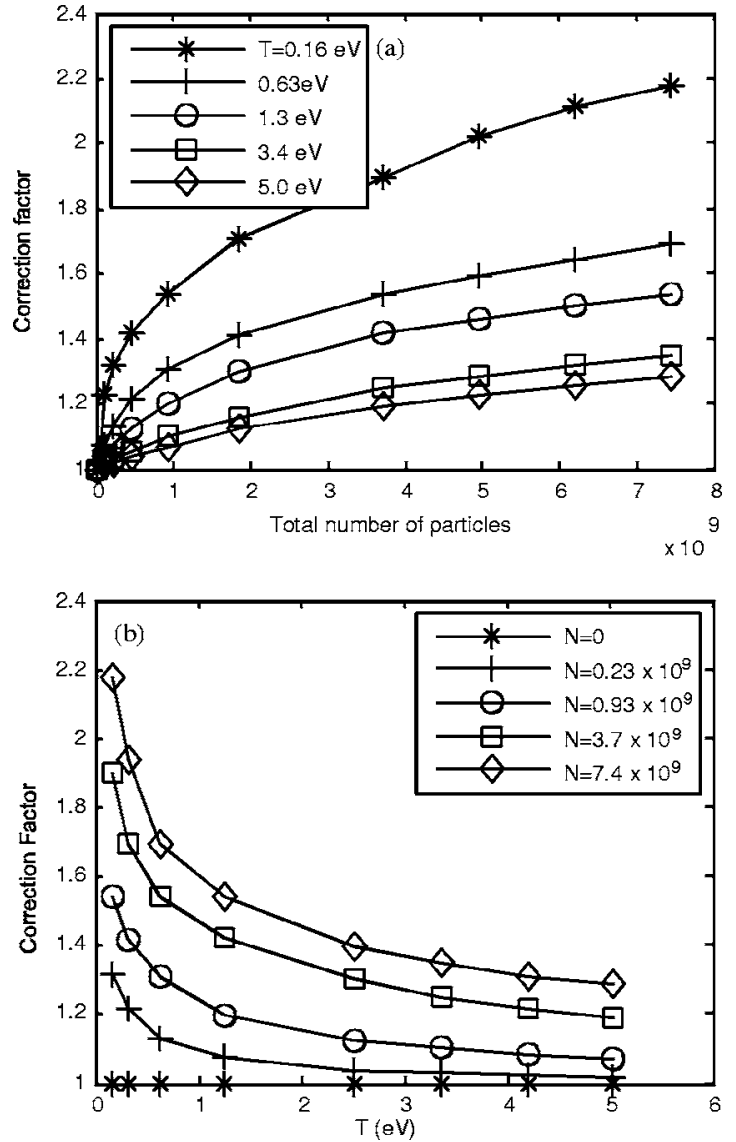

FIG. 9. (a) Theoretical curves of the correction factor as a function of total number of particles. Each curve is for the indicated temperature. (b) Theoretical curves of the correction factor as a function of temperature. Each curve is for the indicated total number of particles.

would occur over a larger voltage range, decreasing the magnitude of $d \phi_{p} / d \phi_{r}$. Each of these curves can be approximated by a power-law relation of the form

$$
\langle c\rangle=c_{0} n^{p}+1,
$$

where both $c_{0}$ and $p$ are functions of temperature. For example, at the lowest temperature, $T=0.16 \mathrm{eV}, c_{0}=0.91$, and $p=0.38$. At the highest temperature, $T=5.0 \mathrm{eV}, c_{0}=0.18$, and $p=0.68$. Part (b) of the figure shows curves of the correction factor as a function of temperature at several values of the total number of electrons in the plasma. By calculating these curves for different density profiles, we have found that they scale as total particle number rather than central density. Each of these curves can also be approximated by a power law of the form of Eq. (13). With $1.1 \times 10^{8}$ particles, $c_{0}=0.023$ and $p=-0.83$. With $7.4 \times 10^{9}$ particles, $c_{0}=0.40$ and $p=-0.40$.

There is a small effect of temperature profile on this correction factor. When we calculated the correction factor for both a plasma with a uniform temperature and the plasma with the large temperature gradient shown in Sec. V, we found a variation of about 5\% in the value of the correction factor. This is small enough not to be particularly significant in the experimental measurement.

The exact curves shown here are specific to our geometry. They would need to be recalculated for plasmas of dif- ferent lengths and different confinement ring lengths. For example, with longer rings this effect is somewhat less important, but not ignorable. The value of $\langle c\rangle-1$ is about $2 / 3$ as large for a $10 \mathrm{~cm}$ confinement ring as it is for a $2.5 \mathrm{~cm}$ ring. For the case of the data taken at $125 \mathrm{~ms}$ shown above, a $2.5 \mathrm{~cm}$ ring has a correction factor of about 1.3. With a $10 \mathrm{~cm}$ confinement ring the correction factor would be about 1.2. Longer rings have a smaller contribution from $d \phi_{p} / d \phi_{r}$ (the bulk of the plasma is kept farther from the potential peak) but a slightly larger contribution from $d \phi_{c} / d \phi_{r}$ (the plasma has to expand more before it can escape). Even at zero plasma loss the correction factor for a $10 \mathrm{~cm}$ ring is a little below 1.1. Note that with a longer ring the adiabatic expansion of the plasma during the slow dump becomes important. ${ }^{14}$ With a $10 \mathrm{~cm}$ ring length and a $4 \mathrm{~cm}$ ring radius, the adiabatic expansion and this correction factor are of roughly equal size and opposite in direction.

To verify these correction factors, we have compared these theoretical results with measurements of evaporation temperature and equilibrium temperature calculation over a range of temperatures from 4 to $0.3 \mathrm{eV}$ (obtained by an appropriate time delay in our cooling plasma) and total particle number from $5 \times 10^{9}$ to $1 \times 10^{9}$. The theoretical correction factors always made the two agree within the experimental error.

Since these correction factors were calculated using an equilibrium calculation and the evaporation temperature measurement is a dynamic (albeit slow) process, we verified the results using RATTLE. We simulated a slow dump and analyzed the charge that came out as a function of time. To avoid the extra evaporation problem mentioned in Sec. V, we did runs with increasing numbers of simulation particles until the results stopped changing significantly. The results of the RATTLE runs were consistent with the equilibrium calculations and they verified that adiabatic expansion was insignificant for our end rings, but that it would be significant for longer end rings. For $10 \mathrm{~cm}$ rings, the evaporation temperature without any corrections was correct within a few percent because of the offsetting effects of the adiabatic expansion and the effects discussed here.

\section{CONCLUSIONS}

With a short plasma or short confinement rings, it is not possible to make the assumptions that Eggleston made in his paper for finding the temperature profile in a non-neutral plasma in a Malmberg-Penning trap. This can be overcome by using a modified equilibrium calculation to find the mapping between the confinement ring potential and the potential hill that the plasma must overcome. Eggleston's shortcut evaporation method of finding the central temperature is found to need a correction factor even when only a small fraction of the central plasma has been lost. The size of the correction factor depends on the total number of particles in the plasma, the temperature of the plasma, and the fraction of the plasma that is used for the measurement. For a very low number of particles in the plasma, the correction factor approaches 1, but for densities, temperatures, and geometries typical of these experiments the correction factor ranges 
from 1 plus a few percent up to a factor of 2 . Typical correction factors for our plasmas are in the range 1.2-1.5. The proper correction factor can be calculated using the equilibrium techniques described in this paper.

\section{ACKNOWLEDGMENTS}

We appreciate the support of Brigham Young University for equipment and supporting staff. We also appreciate helpful discussions with Ross Spencer and Grant Mason in understanding some of our results.

${ }^{1}$ J. S. deGrassie and J. H. Malmberg, Phys. Rev. Lett. 39, 1077 (1977).

${ }^{2}$ J. H. Malmberg and C. F. Driscoll, Phys. Rev. Lett. 44, 654 (1980).

${ }^{3}$ G. W. Hart, Phys. Fluids B 3, 2987 (1991). Although not specifically mentioned in the paper, the data shown here were taken with concentric rings.
${ }^{4}$ M. D. Tinkle, R. G. Greaves, C. M. Surko, R. L. Spencer, and G. W. Mason, Phys. Rev. Lett. 72, 352 (1994).

${ }^{5}$ A. J. Perrung and J. Fajans, Rev. Sci. Instrum. 64, 52 (1993).

${ }^{6}$ R. L. Spencer, S. N. Rasband, and R. R. Vanfleet, Phys. Fluids B 5, 4267 (1993).

${ }^{7}$ J. H. Malmberg and J. S. deGrassie, Phys. Rev. Lett. 35, 577 (1975).

${ }^{8}$ A. W. Hyatt, C. F. Driscoll, and J. H. Malmberg, Phys. Rev. Lett. 59, 2975 (1987).

${ }^{9}$ F. Anderegg, N. Shiga, D. H. E. Dubin, C. F. Driscoll, and R. W. Gould, Phys. Plasmas 10, 1556 (2003).

${ }^{10}$ D. L. Eggleston, C. F. Driscoll, B. R. Beck, A. W. Hyatt, and J. H. Malmberg, Phys. Fluids B 4, 3432 (1992).

${ }^{11}$ J. D. Moody and C. F. Driscoll, Phys. Plasmas 2, 4482 (1995).

${ }^{12}$ J. K. Jennings, R. L. Spencer, and K. C. Hansen, Phys. Plasmas 2, 2630 (1995); G. W. Mason, R. L. Spencer, and J. A. Bennett, ibid. 3, 1502 (1996).

${ }^{13}$ A. W. Hyatt, Ph.D. thesis, University of California, San Diego, 1988.

${ }^{14}$ A. W. Hyatt, C. F. Driscoll, and J. H. Malmberg, Phys. Rev. Lett. 59, 2975 (1987). 